\title{
SYMPLOCOS STRIATA (SYMPLOCACEAE), UNA ESPECIE NUEVA DE LA VERTIENTE CARIBE DE COSTA RICA
}

\author{
RicARdo Kriebel ${ }^{1} \&$ NeLSON ZAMORA ${ }^{1,2}$ \\ ${ }^{1}$ Instituto Nacional de Biodiversidad (INBio), apdo. 22-3100, Santo Domingo, Heredia, Costa Rica \\ ${ }^{2}$ Organización para Estudios Tropicales (OET), apdo. 676-2050, San Pedro, Costa Rica
}

\begin{abstract}
Symplocos striata, a new species endemic to the Caribbean slope of Costa Rica is described, illustrated and compared to its closest relative. Diagnostic characters of the new taxon include densely hirsute apical branches, serrate leaf margins, sparsely to moderately hirsute abaxial leaf surface, conspicuously reticulate lamina, inflorescence axis short or absent, flowers with apically subulate and abaxially densely sericeous calyx lobes and big, 4-locular fruits with a conspicuously striate endocarp. Symplocos striata is close to S. povedae; differences between these two species are enumerated in a key.

Resumen. Symplocos striata, una nueva especie endémica en la vertiente caribe de Costa Rica, se describe, ilustra y compara con la especie más cercana. Caracteres diagnósticos del nuevo taxon incluyen ramitas distales denso-hirsutas, margen de la lámina aserrado, envés esparcida a moderadamente hirsuto, eje de la inflorescencia corto o ausente, flores con los lóbulos del cáliz apicalmente subulados y abaxialmente densoseríceos y frutos grandes, tetraloculares, con el endocarpo conspicuamente estriado. Symplocos striata es afín a $S$. povedae; diferencias entre ambas especies se enumeran en una clave.
\end{abstract}

Palabras Clave / KeY words: Symplocaceae, Symplocos striata, Costa Rica

La familia Symplocaceae se distribuye en las zonas tropicales y subtropicales de América, así como en el sur y el este de Asia y en Australia; su único género, Symplocos Jacq., cuenta con aproximadamente 250 a 300 especies (Almeda 1982). La mayoría de las especies prefieren hábitats poco alterados; la continua dismi-nución de éstos hace difícil la localización de estas plantas en el campo. Por el hábito arbóreo usualmente pequeño, la floración atractiva y abundante, los frutos apetecidos por la fauna silvestre y la disminución de las poblaciones de algunas especies, recomendamos el uso de especies de Symplocos para el embelle-cimiento de áreas urbanas y jardines.

De las 22 especies mesoamericanas (L. Kelly \& Almeda, en prensa), 12 habitan en Costa Rica (incluida la descrita aquí), seis de éstas son hasta ahora endémicas ( $S$. naniflora L. Kelly \& Almeda, $S$. oreophila Almeda, S. povedae Almeda, S. retusa Kriebel, González \& Alfaro, S. striata Kriebel \& Zamora y $S$. tribracteolata Almeda) y siete han sido descritas en las últimas tres décadas (Almeda 1982, Kelly \& Almeda 2002, Kriebel et al. 2004, McPherson 1988). Durante la revisión de la familia
Symplocaceae para el proyecto del Manual de Plantas de Costa Rica surgió una especie muy distintiva, que se describe a continuación.

Symplocos striata Kriebel \& N. Zamora, sp. nova

TIPO: Costa Rica. Heredia: Finca La Selva, Puerto Viejo de Sarapiquí, loop trail, 6 Jul 1979, J. Sperry 817 (Holotipo: DUKE, isotipos: CAS, CR, INB).

FIG. 1.

A Symplocoe povedae affinis, sed lamina non bullata, calycis corollaeque lobis minoribus, corolla cum octo vel novem lobis, fructo tetraloculari, endocarpo conspicue striato differt.

Árbol 10-15 m; yemas vegetativas y ramitas jóvenes cilíndricas, densamente hirsutas con tricomas de 1-2.25 mm. Pecíolos 3-9 mm de largo, hirsutos; lámina foliar 9.5-21 x 3.5-7.2 cm, elíptica a elípticoobovada, cartácea, margen aserrado, ápice acuminado, el acumen 1-2.3 cm, base angostamente truncada, con 7 a 11 pares de nervios secundarios, reticulada en ambas caras, haz liso a levemente abollado, esparci- 


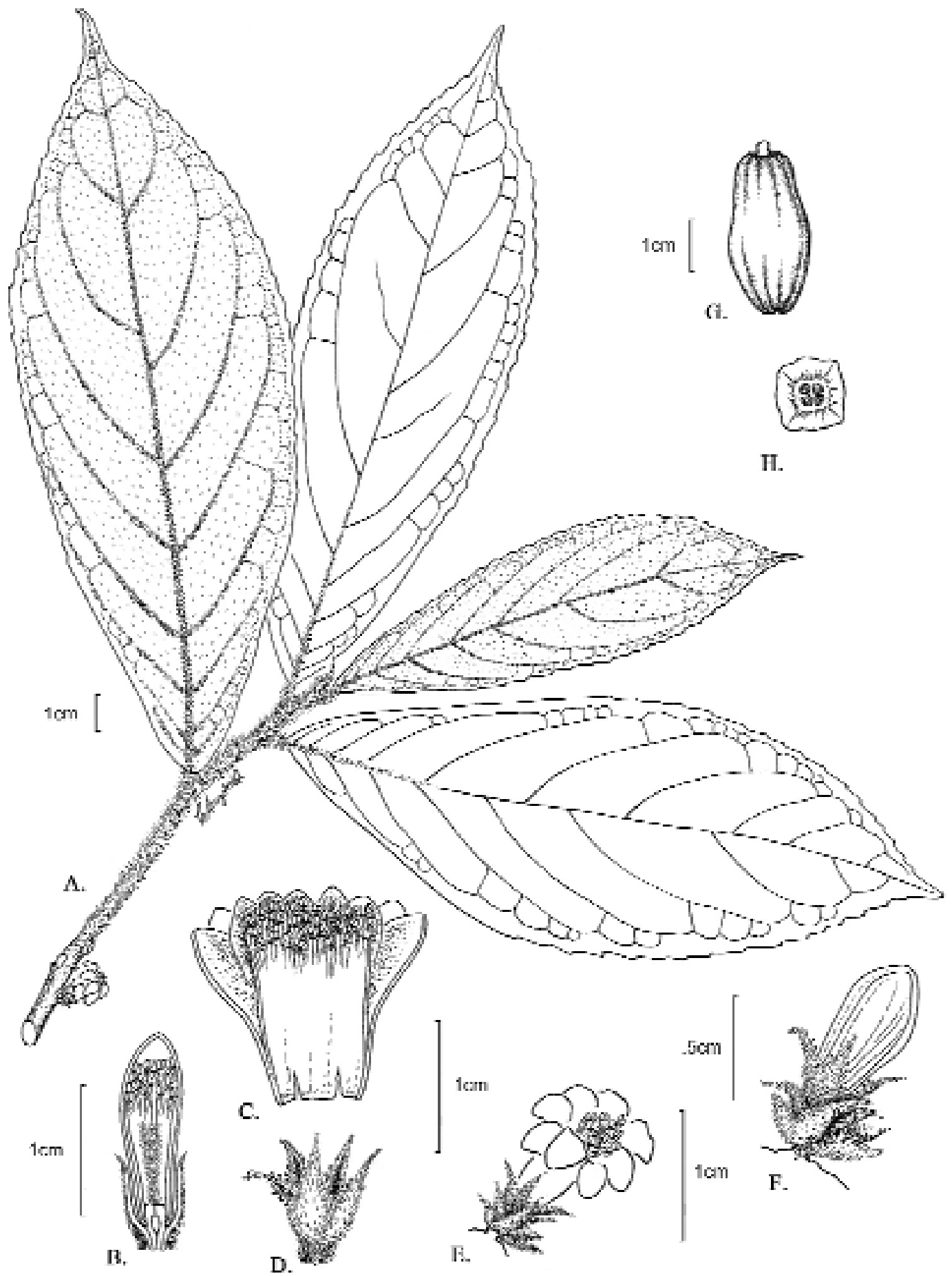

Fig. 1. Symplocos striata Kriebel \& N. Zamora. A. Hábito. B. Corte longitudinal de un botón floral. C. Corte longitudinal de la corola. D. Cáliz. E. Cáliz y corola. F. Botón floral. G. Corte transversal del fruto. H. Fruto. A-F, Hammel 9255 (LSCR); G y H, Hartshorn 1599 (LSCR, DUKE). 
damente estriguloso en hojas jóvenes a glabrescente en hojas maduras, envés moderadamente hirsuto en las venas principales y esparcidamente hirsuto en la superficie, los tricomas lisos, de 0.5-1.25 mm. Inflorescencia un racimo axilar de 1-5 mm, la hoja que la subtiende frecuentemente caduca; pedicelo ausente o hasta $0.5 \mathrm{~mm}$; brácteas y bracteolas numerosas, de 3-5 x 1-2 mm, triangulares a triangular-ovadas, con el ápice largo-acuminado, seríceas abaxialmente, persistentes; cáliz pentalobado, el tubo 1-2 mm, seríceo abaxialmente, los lóbulos 4-5 x 1-1.5 $\mathrm{mm}$, angostamente lanceolados, seríceos abaxialmente, el ápice subulado, el margen ciliado con glándulas estipitadas esparcidamente entremezcladas, o ausentes; corola 10-16 mm, con 8 ó 9 lóbulos, pétalos biseriados, oblongos, glabros, connados entre sí 6-9 $\mathrm{mm}$ y adnados al tubo estaminal $8-11 \mathrm{~mm}$; estambres multiseriados, filamentos $13-16 \mathrm{~mm}$, connados entre sí $2 / 3$ a 3/4 de su longitud. Ovario aparentemente seríceo apicalmente; estilo ca. $14 \mathrm{~mm}$, glabro. Frutos 30-35 x 12-16 mm, azules, elipsoides a oblongos, glabros, tetraloculares, el perímetro del endocarpo finamente estriado, (excepto en las cuatro esquinas, con una estría conspicua, $c a .2 \mathrm{~mm}$ de largo, que atraviesa prácticamente el endocarpo).

DistRIBUCIÓN. Hasta ahora endémica en Costa Rica; se ha recolectado en bosque muy húmedo de tierras bajas entre 50 y 100 (-400) m de elevación en la zona norte o septentrional de la vertiente caribe (Boca Tapada, Pital, San Carlos) hacia el sur hasta la Estación Biológica La Selva, Sarapiquí, y las Llanuras de Santa Clara, Pococí.

FENOLOGía. Se ha recolectado con flores en julio y con frutos en agosto y diciembre.

ETimología. El epíteto striata hace alusión al endocarpo conspicuamente estriado.

La primera recolecta conocida de $S$. striata es un espécimen con frutos hallado en 1974 por Gary S. Hartshorn en Finca La Selva, Sarapiquí, Heredia. Desde entonces se han realizado cuatro recolectas con flores, lo que ha permitido aquí la descripción formal de esta especie. Symplocos striata se caracteriza por sus hojas relativamente grandes, con el margen aserrado, la lámina conspicuamente reticulada en ambas caras, las yemas vegetativas y las ramitas distales denso-hirsutas, la inflorescencia reducida, los lóbulos del cáliz con el ápice subulado, denso-seríceos abaxialmente, los frutos grandes y el perímetro del endocarpo conspicuamente estriado. Además, $S$. striata es similar a $S$. povedae; ambas sobresalen del resto de las especies costarricenses especialmente por las inflorescencias reducidas, los lóbulos del cáliz densamente seríceos abaxialmente y con el ápice acuminado a subulado. Ambas se diferencian con la siguiente clave:

1 Corola con 10 ó 11 lóbulos, rosada con el tubo blanco y secando rojizo a anaranjado, $1.6-2.5 \mathrm{~cm}$ en antesis; pétalos 5-8 mm de ancho; lóbulos del cáliz 4-8 $\mathrm{mm}$ de largo, el ápice acuminado; lámina foliar conspicuamente abollada; fruto trilocular con el perímetro del endocarpo liso a ondulado; > $1400 \mathrm{~m}$ de elevación ...... S. povedae

1a Corola con 8 ó 9 lóbulos, blanca y secando oscuroamarillento, 1-1.6 cm en antesis; pétalos 2-4 $\mathrm{mm}$ de ancho; lóbulos del cáliz 4-5 mm de largo, el ápice subulado; lámina foliar levemente abollado-reticulada; fruto tetralocular con el perímetro del endocarpo conspicuamente estriado; < $100(-400)$ m de elevación ....... S. striata

En la región norte del país, Symplocos striata se ha observado creciendo simpátricamente con $S$. naniflo$r a$ L. Kelly \& Almeda. Hemos observado que plantas jóvenes de S. naniflora se asemejan más a S. striata que a los árboles maduros, debido a que tienden a tener hojas más pubescentes y reticuladas; pero ambas especies se diferencian fácilmente porque $S$. naniflora posee hojas más pequeñas, ramitas y hojas glabras a seríceas o esparcido-pilosas, flores muy pequeñas, con los lóbulos del cáliz glabros y con el ápice redondeado y frutos más pequeños.

Paratipos. Costa Rica. Alajuela: San Carlos, Llanuras de San Carlos, Pital, Finca Hiloba, $18 \mathrm{~km} \mathrm{~N}$ de Boca Tapada, $10^{\circ} 46^{\prime} \mathrm{N}, 84^{\circ} 11^{\prime} \mathrm{W}, 50 \mathrm{~m}, 1$ abr 1995, $Q$. Jiménez \& R. Quesada 1739 (INB); loc. cit., Pital, Boca Tapada, Finca San Jorge, $10^{\circ} 42^{\prime} \mathrm{N}, 84^{\circ} 10^{\prime} \mathrm{W}, 50 \mathrm{~m}, 20$ jun 1996, A. Rodríguez et al. 1158 (CR, INB, MO); loc. cit. Pital, Boca Tapada, Finca Aserradero San Jorge, 10 $44^{\prime} \mathrm{N}$, $84^{\circ} 10^{\prime} \mathrm{W}, 100 \mathrm{~m}, 21$ ene 1996, N. Zamora \& A. Zeledón 2363 (CR, INB, MO). HEREDIA: Finca La Selva, the OTS Field Station on the Río Puerto Viejo just $\mathrm{E}$ of its junction with the Río Sarapiquí, $100 \mathrm{~m}, 19$ Jul 1980, $B$. Hammel 9255 (LSCR, Herbario de la Estación Biológica La Selva); Finca La Selva, Puerto Viejo de Sarapiquí, 
Vargas clearing along the west boundary, $10^{\circ} 26^{\prime} \mathrm{N}$, 8401'W, 5 Aug 1974, G.S. Hartshorn 1599 (LSCR, DUKE); Finca La Selva, Puerto Viejo de Sarapiquí, El Surá near Taconazo Creek, 9 Jul 1979, J. Sperry 876 (DUKE). LIMÓN: Cantón de Pococí, Llanura de Santa Clara, Chiporrisito, $10^{\circ} 36^{\prime} 10$ ?N, 834' 20 ?W, 400 m, 30 ene 1995, A. Rodríguez 513 (CR, INB, USJ).

\section{LITERATURA CITADA}

Almeda, F. 1982. Three new Costa Rican species of Symplocos (Symplocaceae). Bull. Torrey Bot. Club. 109: 318-324.
Kelly, L. \& Almeda, F. 2002. Three new species of Symplocos (Symplocaceae) from Panama and Costa Rica. Novon 12: 369-374.

Kelly, L. \& Almeda, F. Symplocaceae. In: Davidse, G., Sousa S., M. \& Chater, A.O. (eds.). Flora Mesoamericana. Missouri Bot. Gard. Press (manuscrito inédito).

Kriebel, R., Gonzalez, J. \& Alfaro, E. 2004. Symplocos retusa (Symplocaceae), una nueva especie de Costa Rica. Lankesteriana 4(1): 57-59.

McPherson, G. 1988. New and noteworthy taxa from Panama. Ann. Missouri Bot. Gard. 75: 1-34. 